\title{
Potential Interference of Oil Vehicles on Genital Tubercle Development during the Fetal Period in ICR Mice
}

\author{
Yasushi Nishioka, ${ }^{a \dagger}$ Kazuki Tamai, ${ }^{a}$ Masanari Onda, ${ }^{a}$ Youhei Hiromori, ${ }^{a, b}$ Tomoki Kimura,${ }^{c}$ \\ Jianying Hu, ${ }^{d}$ Hisamitsu Nagase, ${ }^{a}$ and Tsuyoshi Nakanishi*, ${ }^{*}$ \\ ${ }^{a}$ Laboratory of Hygienic Chemistry and Molecular Toxicology, Gifu Pharmaceutical University; 1-25-4 Daigaku- \\ nishi, Gifu, Gifu 501-1196, Japan: ${ }^{b}$ Faculty of Pharmaceutical Sciences, Suzuka University of Medical Science; \\ 3500-3 Minamitamagaki, Suzuka, Mie 513-8670, Japan: ${ }^{c}$ Department of Life Science, Faculty of Science and \\ Engineering, Setsunan University; 17-8 Ikedanaka-machi, Neyagawa, Osaka 572-8508, Japan: and ${ }^{d}$ College of \\ Urban and Environmental Sciences, Peking University; No. 5 Yiheyuan Road, Haidian District, Beijing 100871, China. \\ Received October 15, 2017; accepted October 30, 2017
}

Corn oil, sesame oil, and $\mathbf{1 0 \%}$ ethanol in corn oil are commonly used as dosing vehicles in toxicology studies. Since these vegetable oils contain bioactive compounds, it is important for toxicology studies to characterize the toxicities of the dosing vehicles themselves. It has been recently proposed that the width of the genital tubercle (GT), the dorsal-ventral length (D-V length) of the GT, and urethral tube closure in mouse fetuses can be used as novel markers for monitoring sexual development in mice. However, how these parameters are influenced by the dosing vehicles themselves remains unclear. Therefore, we evaluated the effects of corn oil, sesame oil, and $\mathbf{1 0 \%}$ ethanol in corn oil on GT width, D-V length, and GT morphology in ICR mice. Our results showed that all three vehicles influenced GT width and D-V length, but not GT morphology, suggesting that the effects of dosing vehicles themselves might need to be considered when GT width or D-V length is used as a parameter to evaluate the effects of chemicals on GT development.

Key words corn oil; sesame oil; external genitalia; anogenital distance; dosing vehicle; sex difference

Corn oil and sesame oil are commonly employed as vehicles for lipophilic chemicals in in vivo toxicological studies, and $10 \%$ ethanol in corn oil is occasionally used as a vehicle for chemicals with low solubility in oils. Since the control groups in such studies are treated with the vehicle only, the effects of the vehicle itself on animals should be clarified. Several reports have shown that vegetable oils themselves exert certain biological effects on animals. Oral exposure of rats to corn oil during the premating period and the perinatal period decreased the viability of neonates and induced severe lesions in the proximal tubules of the maternal kidney. ${ }^{1)}$ In immature rats, corn oil by subcutaneous injection decreased absolute uterine weights, relative ovarian weights, and relative testis weights compared to the weights in groups not treated with corn oil. ${ }^{2)}$ Sesame oil also decreased relative testis weights. ${ }^{2)}$ These reports suggest that vegetable oils can be a confounding factor in reproductive toxicity studies.

Corn oil and sesame oil are often utilized as vehicles for androgens and anti-androgenic agents such as flutamide and finasteride, exposure to which during fetal development induces malformations of the external genitalia. ${ }^{3-6)}$ One of the most common parameters used to assess anti-androgenic effects of chemicals on fetuses is the anogenital distance (AGD), which is the distance from the genital tubercle (GT) to the anus. AGD shows sexual dimorphism in mammals, including humans and rodents, with AGD in males being larger than that in females. Recently, Suzuki et al. proposed three novel parameters in addition to AGD-GT width, dorsal-ventral length of the GT (D-V length), and urethral tube closurethat also show sexual dimorphism and whose male phenotypes

${ }^{\dagger}$ Present address: Toxicology Division, Institute of Environmental Toxicology; 4321 Uchimoriya-machi, Joso, Ibaraki 303-0043, Japan. are changed into female-like phenotypes through exposure to anti-androgens. ${ }^{7)}$

In the current study, we evaluated how administration of corn oil, sesame oil, or $10 \%$ ethanol in corn oil to maternal mice influenced these three new parameters as well as AGD (the typical parameter used for assessing development of the external genitalia) in fetal mice. Based on our assessments of AGD, GT width, D-V length, and urethral tube closure, we discuss the effects of each vehicle on androgen-associated development and the suitability of these new parameters for assessing adverse effects of chemicals on development of external genitalia.

\section{MATERIALS AND METHODS}

Chemicals Corn oil, sesame oil, and paraformaldehyde were purchased from Nacalai Tesque (Kyoto, Japan). Ethanol was obtained from Kanto Chemical Co., Inc. (Tokyo, Japan). All other chemicals were of guaranteed reagent grade.

Animals Time-mated ICR mice at gestation day (GD) 6-8 (vaginal plugs noted=GD 0) were purchased from SLC Inc. (Shizuoka, Japan). Mice were housed in a room maintained at $23 \pm 2{ }^{\circ} \mathrm{C}$ with $50 \pm 10 \%$ humidity and a $12: 12$-h light-dark cycle (lights on from 8:00 a.m. to 8:00 p.m.) and were fed a diet of standard mouse chow (CE-2; CLEA, Tokyo, Japan). Food and water were provided ad libitum. All animal care and handling procedures were approved by the Institutional Animal Care and Use Committee of Gifu Pharmaceutical University. All efforts were made to minimize both the number of animals used and the pain and distress they experienced.

Maternal Treatment and Fetus Sampling Dams were randomly divided into four groups (4-6 dams per group): corn oil group (CO group), sesame oil group (SO group), or $10 \%$ 


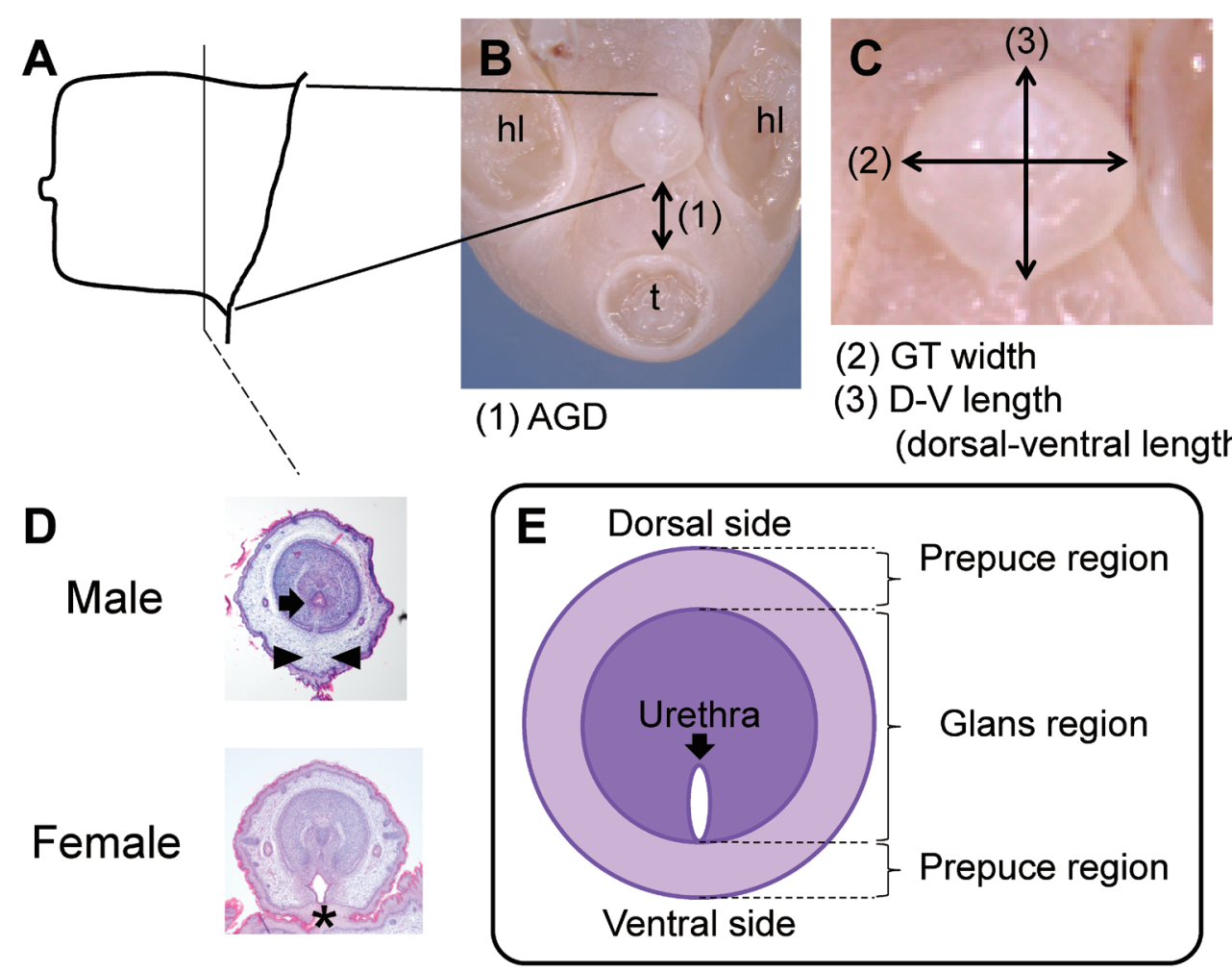

Fig. 1. Parameters of Genital Sex Differentiation

(A) Illustration of a sagittal view of the genital tubercle (GT). (B) The distance from the GT to the anus is defined as AGD (1). (C) GT width (2) and D-V length (3) are defined in the coronal view of the GT. (D) Histological sections of the GT in male and female mice. The black arrow and opposing arrowheads represent the urethra in the glans region and the fused prepuce, respectively. The asterisk indicates the non-tubular urethra or urethra outside of the glans regions. (E) Illustration of morphological structure of the GT. A male GT typically has the urethra in the glans region; the female urethra typically forms outside of the glans region and opens outward. hl, hind limb; t, tail.

ethanol in corn oil group (E-CO group). Dams were weighed, and the respective treatment was subcutaneously administered at $10 \mathrm{~mL} / \mathrm{kg}$ bodyweight daily from GD 9 to GD 17 . On GD 18 , dams were killed by inhalation of diethyl ether, and the fetuses and placentas were collected and weighed. The fetuses were fixed in $4 \%$ paraformaldehyde in phosphate-buffered saline overnight at $4^{\circ} \mathrm{C}$.

Male sex of a fetus was determined by detection of the Sry gene (accession number NC_000087.7) by PCR analysis using fetal tail DNA. Briefly, mouse DNA was amplified by 35 cycles $\left(30 \mathrm{~s}\right.$ at $95^{\circ} \mathrm{C}, 30 \mathrm{~s}$ at $60^{\circ} \mathrm{C}, 30 \mathrm{~s}$ at $\left.72^{\circ} \mathrm{C}\right)$ using Quick Taq HS dye mix (Toyobo, Osaka, Japan). The forward and reverse primers for Sry were 5'-CAGCAGAAT CCC AGCATGCAA$3^{\prime}$ and 5'-GGT TCC TGT CCC ACT GCA GAA-3', respectively. The amplified 250-bp PCR product was detected by agarose gel electrophoresis.

Measurement of AGD, GT Width, and D-V Length AGD, GT width, and D-V length were evaluated in the fixed fetuses. Micrographic images were taken with a stereomicroscope (Leica M205 A; Leica Microsystems, Wetzlar, Germany), and the three distances were measured using the accessory software (LAS V3.8; Leica Microsystems) (Fig. 1). Since AGD, GT width, and D-V length are also influenced by body size, these parameters were normalized by the cube root of body weight, and the normalized values are referred to here as anogenital index (AGI), ${ }^{8}$ relative GT width, and relative D-V length, respectively. For the fetus data (fetal weight, placental weight, AGD, AGI, GT width, relative GT width, D-V length, and relative $\mathrm{D}-\mathrm{V}$ length), male and female group means were calculated by using the means of each sex in each litter.

GT Sections After the AGD and GT were measured, the GT was excised, dehydrated in increasing concentrations of ethanol, cleared with xylene, and embedded in paraffin. Sections $(5 \mu \mathrm{m})$ were then deparaffinized with xylene and rehydrated with ethanol and water. The rehydrated sections were stained using Mayer's hematoxylin (Wako Pure Chemical Industries, Ltd., Osaka, Japan) and 1\% eosin Y solution (Muto Chemical, Tokyo, Japan) and examined under a microscope (BX51; Olympus, Tokyo, Japan).

Statistical Analysis All data were analyzed by using SPSS 15.0 J software (SPSS, Chicago, IL, U.S.A.). Values are expressed as mean \pm 1 standard deviation (S.D.). Data containing two groups were analyzed by Student's $t$-test, and data containing four groups were analyzed by one-way ANOVA followed by Dunnett's test. A $p$ value of less than 0.05 was considered significant.

\section{RESULTS AND DISCUSSION}

Pregnancy Parameters, Fetal Weights and Placental Weights There were no significant difference between the saline group and the other three groups in terms of maternal body weights from GD 9 to 18 (just before the maternal animals were killed), litter size, or fetal sex ratio (Table 1).

There was no difference between male fetal weights and female fetal weights in the saline group (Fig. 2A). Male fetal weight was significantly but only slightly heavier than female fetal weight in the SO group; however, no sex differences 
Table 1. Summary of Pregnancy Parameters

\begin{tabular}{|c|c|c|c|c|}
\hline Parameters & Saline & $\mathrm{CO}$ & $\mathrm{SO}$ & $\mathrm{E}-\mathrm{CO}$ \\
\hline Pregnant number & $n=4$ & $n=6$ & $n=5$ & $n=5$ \\
\hline \multicolumn{5}{|c|}{ Dam's weight during pregnancy (g) } \\
\hline GD9 & $39.06 \pm 1.69$ & $39.74 \pm 4.34$ & $40.11 \pm 3.32$ & $40.76 \pm 2.05$ \\
\hline GD10 & $40.23 \pm 1.81$ & $42.11 \pm 3.21$ & $41.72 \pm 2.87$ & $42.61 \pm 1.84$ \\
\hline GD11 & $42.57 \pm 2.67$ & $44.49 \pm 3.21$ & $43.49 \pm 2.76$ & $44.76 \pm 2.02$ \\
\hline GD12 & $44.57 \pm 2.54$ & $47.70 \pm 3.25$ & $46.65 \pm 2.96$ & $47.83 \pm 1.70$ \\
\hline GD13 & $47.65 \pm 3.11$ & $50.17 \pm 3.45$ & $49.41 \pm 2.45$ & $49.78 \pm 2.58$ \\
\hline GD14 & $51.40 \pm 3.27$ & $53.68 \pm 3.90$ & $52.02 \pm 2.74$ & $54.07 \pm 2.00$ \\
\hline GD15 & $56.25 \pm 3.87$ & $57.62 \pm 4.60$ & $56.29 \pm 3.00$ & $58.31 \pm 2.69$ \\
\hline GD16 & $60.37 \pm 4.62$ & $63.42 \pm 5.56$ & $60.60 \pm 3.95$ & $62.58 \pm 3.68$ \\
\hline GD17 & $65.28 \pm 5.42$ & $68.26 \pm 4.78$ & $65.01 \pm 4.26$ & $67.10 \pm 3.12$ \\
\hline GD18 & $70.15 \pm 5.88$ & $73.36 \pm 4.85$ & $68.62 \pm 4.41$ & $71.07 \pm 5.12$ \\
\hline Litter size $^{a)}$ & $13.3 \pm 1.3$ & $13.8 \pm 1.9$ & $11.6 \pm 2.9$ & $13.0 \pm 1.2$ \\
\hline Sex ratio $^{b)}$ & $0.626 \pm 0.177$ & $0.533 \pm 0.110$ & $0.557 \pm 0.085$ & $0.567 \pm 0.122$ \\
\hline
\end{tabular}

Each value represents the means \pm S.D. of 4-6 dams per group. In the statistical analyses, one-way ANOVA followed by Dunnett's test was conducted between the four groups ( $v s$. saline). Significant difference: $p<0.05 . a$ ) Number of live fetuses per litter. $b$ ) Ratio of live male fetuses to total number of live fetuses per litter.

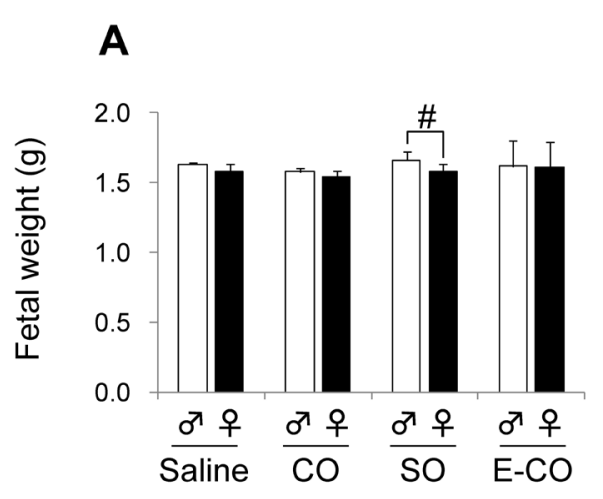

B

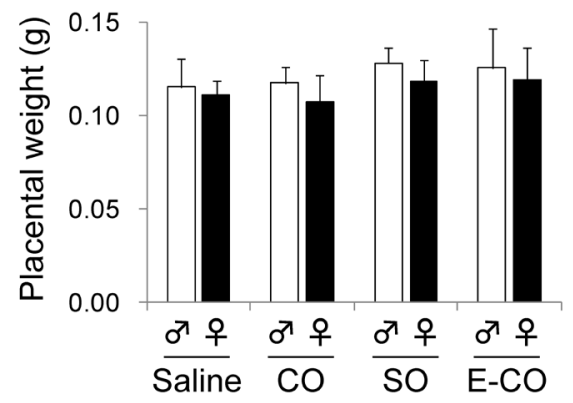

C

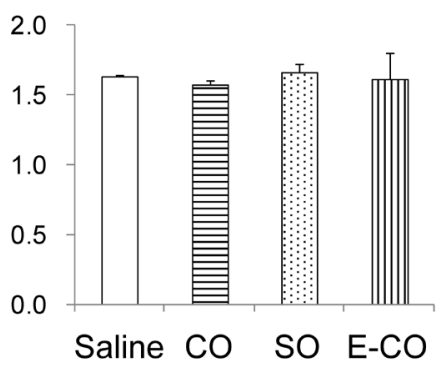

D

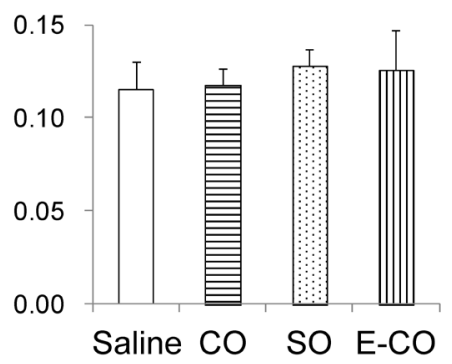

E

Female

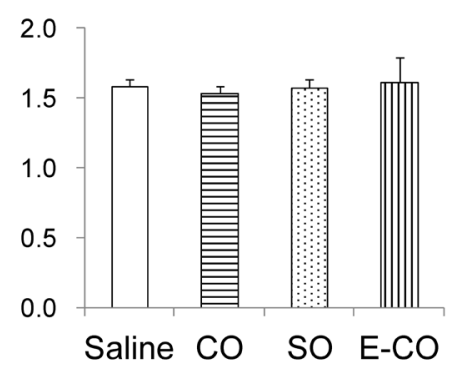

F

Female

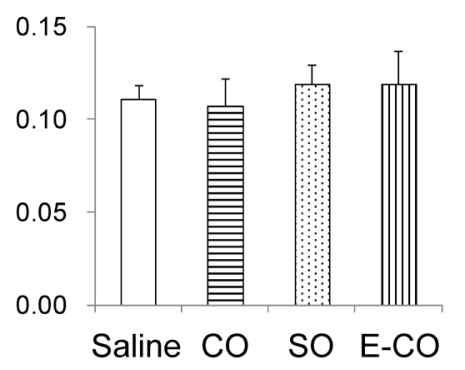

Fig. 2. Effect of Vehicle on Fetal Weight (A, C, E) and Placental Weight (B, D, F)

Values represent means \pm S.D.; $n=4-6$ litters per group. ${ }^{\#} p<0.05$ (Student's $t$-test).

were observed in the $\mathrm{CO}$ group or the E-CO group. Comparison between the vehicle groups showed no significant difference in fetal weights of each sex (Figs. 2C, E). There was also no significant difference between sexes in terms of placental weight in any of the groups treated with each vehicle (Fig. 2B) and no significant difference between the saline group and any of the other vehicle-treated groups of each sex (Figs. 2D, F).

These results suggest that all tested oil vehicles possibly have no influence on AGD, GT width, D-V length or GT morphology due to a change in the fetal and placental weights.

AGD and AGI It is well-known that AGD and AGI are larger in males than in females. The saline group consistently showed male AGD and AGI to be larger than those of females
(Figs. 3A, B). The same sex differences in AGD and AGI were also observed in the other three vehicle-treated groups. In the comparisons between the four groups, there were no significant differences in AGD or in AGI for either males or females (Figs. 3C-F). Therefore, these results indicate that corn oil, sesame oil, and $10 \%$ ethanol in corn oil are not confounding factors in the evaluation of AGD and AGI.

GT Width and Relative GT Width Suzuki et al. reported that in C57BL/6 mice, GT width was larger in males than in females. ${ }^{7)}$ However, we observed no sex difference in GT width or relative GT width in saline-treated ICR mice (Figs. 4A, B). Similarly, there were no sex differences in these parameters in the $\mathrm{SO}$ group or the $\mathrm{E}-\mathrm{CO}$ group. In the $\mathrm{CO}$ 


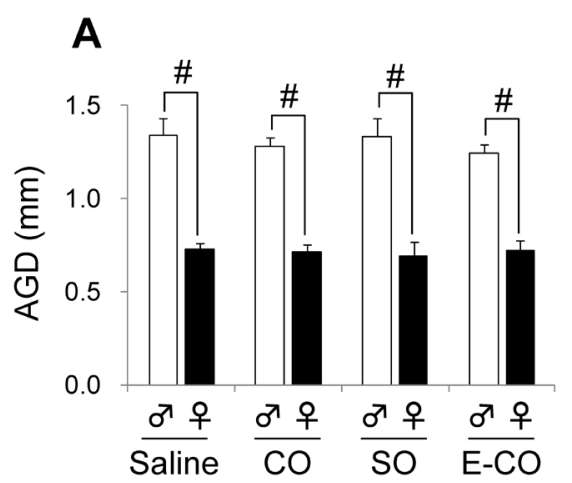

C Male

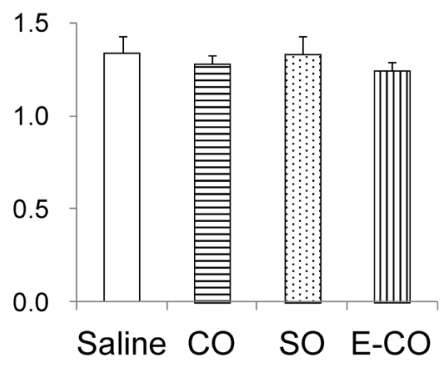

$\mathbf{E}$

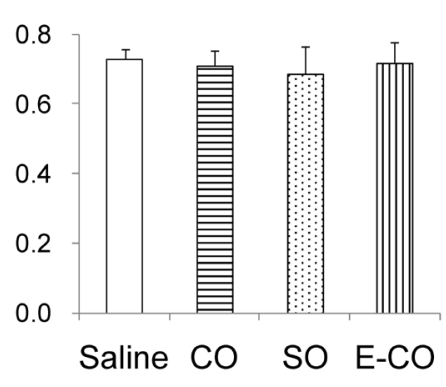

B

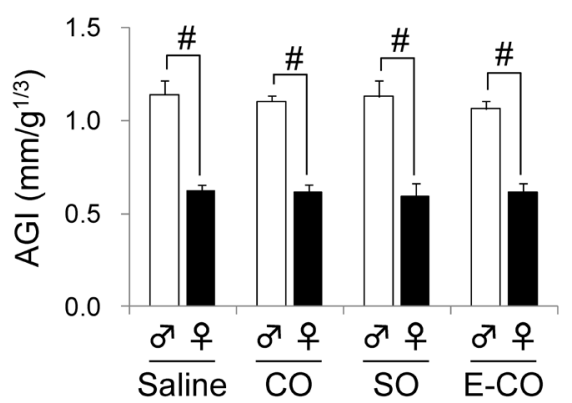

D

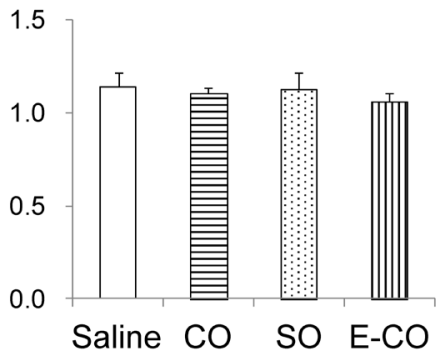

F

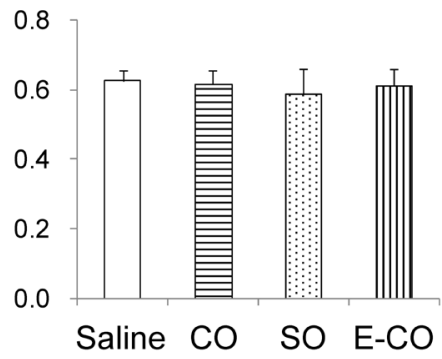

Fig. 3. Effect of Vehicle on AGD (A, C, E) and AGI (B, D, F)

Values represent means \pm S.D.; $n=4-6$ litters per group. ${ }^{*} p<0.05$ (Student's $t$-test).
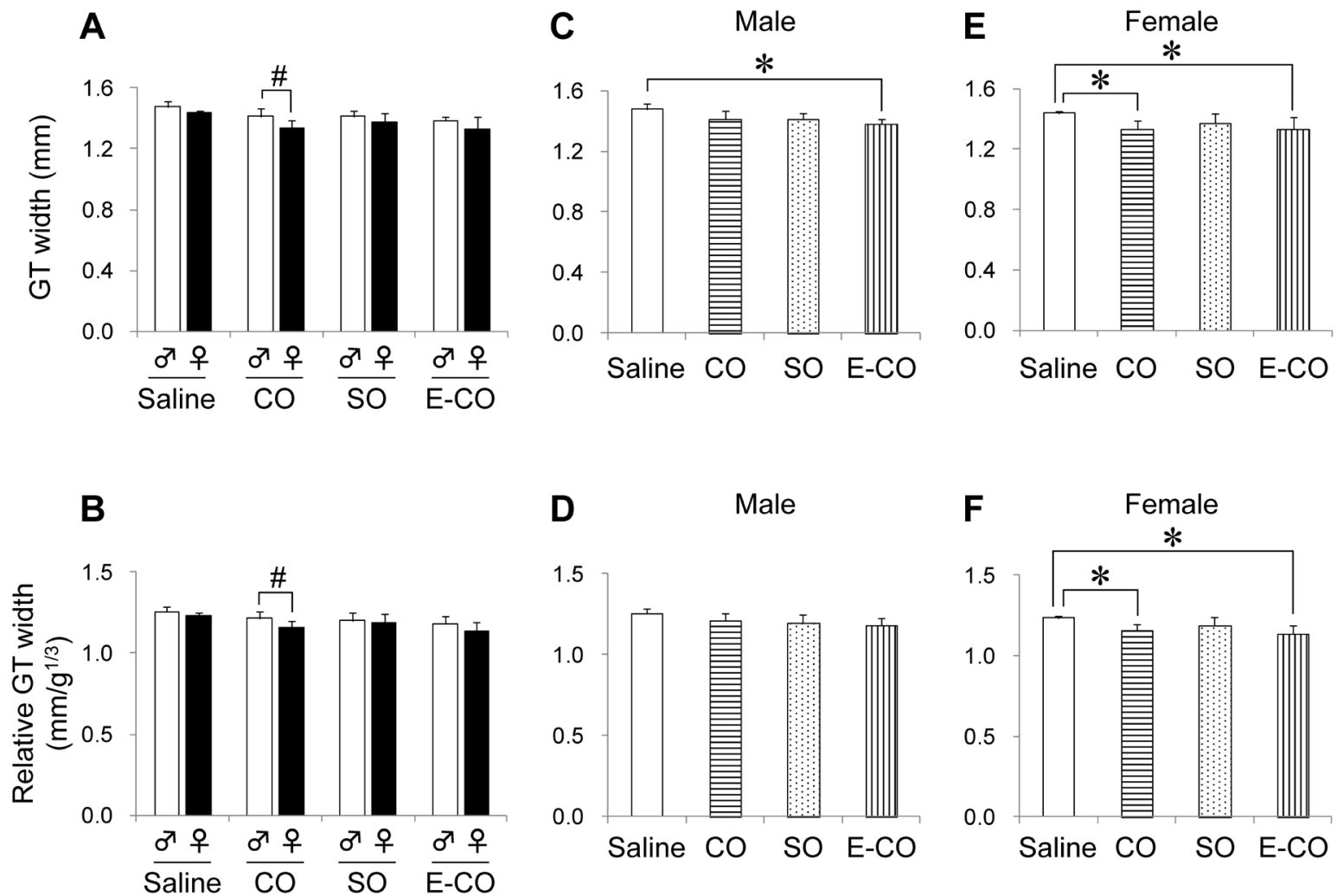

Fig. 4. Effect of Vehicle on GT Width (A, C, E) and Relative GT Width (B, D, F)

Values represent means \pm S.D.; $n=3-6$ litters per group. ${ }^{*} p<0.05$ (Student's $t$-test); ${ }^{*} p<0.05$ (vs. saline group; one-way ANOVA followed by Dunnett's test).

group, however, these parameters were significantly larger in males than in females.

Comparing between vehicles, for males, the GT width in the E-CO group was significantly smaller than that in the saline group (Fig. 4C), whereas the relative GT width in the E-CO group was comparable to that in the saline group (Fig. 
A

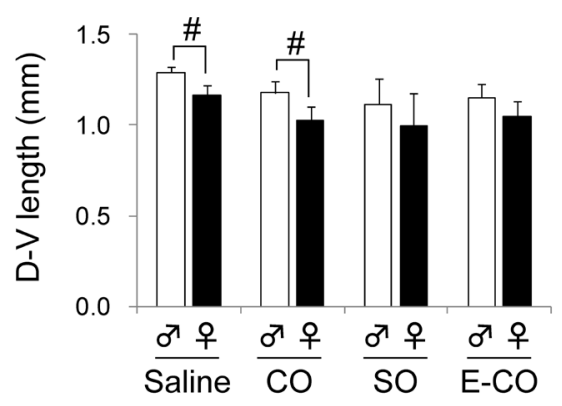

C

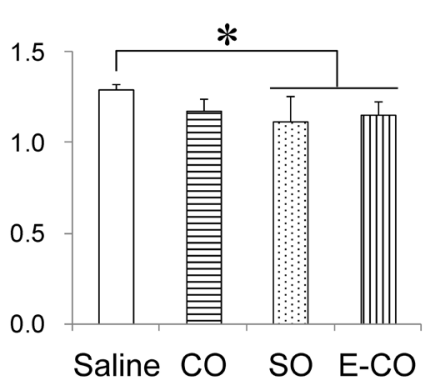

E

Female

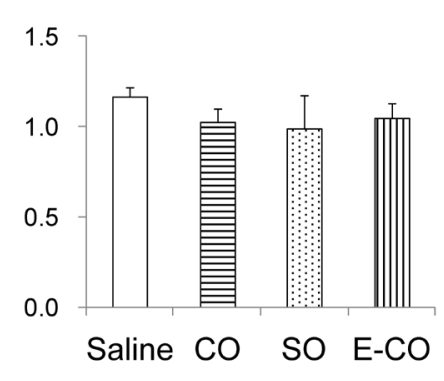

B

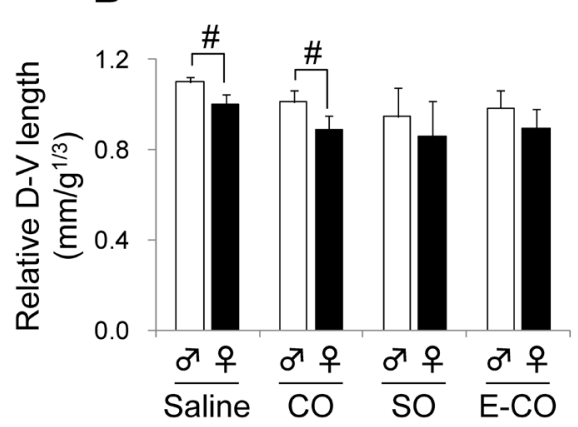

D

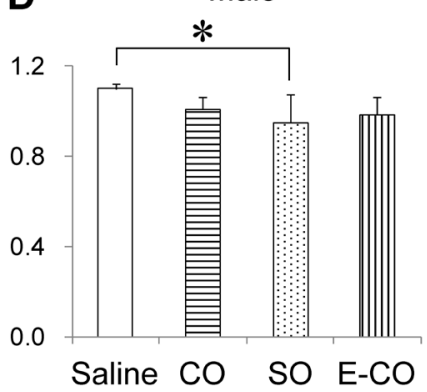

F

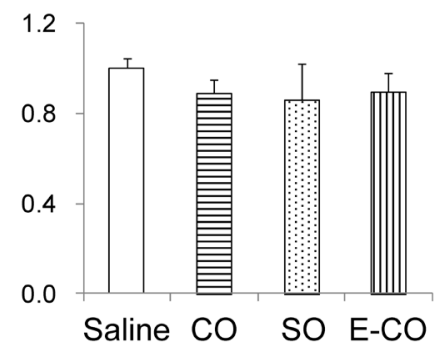

Fig. 5. Effect of Vehicle on D-V Length (A, C, E) and Relative D-V Length (B, D, F)

Values represent means \pm S.D.; $n=3-6$ litters per group. ${ }^{*} p<0.05$ (Student's $t$-test); ${ }^{*} p<0.05$ ( $v s$. saline group; one-way ANOVA followed by Dunnett's test).

\begin{tabular}{|c|c|c|c|c|}
\hline & Saline & $\mathrm{CO}$ & SO & E-CO \\
\hline Male & & & & \\
\hline Female & & & & \\
\hline & & & & \\
\hline
\end{tabular}

Fig. 6. Effect of Vehicle on GT Morphology

Black arrows indicate the urethra within the glans region, and opposing arrowheads indicate fused prepuce. Asterisks indicate a non-tubular urethra or urethra outside of the glans region.

4D). For females, both GT width and relative GT width were significantly smaller in the E-CO group than in the saline group (Figs. 4E, F). In addition, significant reductions in GT width and relative GT width were also observed in females of the $\mathrm{CO}$ group.

These results suggest that maternal exposure to corn oil or $10 \%$ ethanol in corn oil might influence GT width. Furthermore, GT width might also be influenced by differences in mouse strain, rather than only by mouse sex, because results observed with sesame oil-treated ICR mice in our study differed from the results of a previous study in which sesame oil-treated C57BL/6 mice were used as a vehicle control. ${ }^{7)}$ Considering these results together, the GT width in ICR mice might be an unsuitable parameter for assessing sex-specific effects of chemicals using corn oil as a vehicle.

D-V Length and Relative D-V Length In a previous study, the D-V length in sesame oil-treated C57BL/6 mice was used as an index of sex difference. ${ }^{7)}$ Consistent with that study, male fetuses of saline-treated ICR mice had D-V length and relative $\mathrm{D}-\mathrm{V}$ length that were significantly larger than those of female fetuses (Figs. 5A, B). The CO group also showed significant sex differences in these parameters, 
whereas there were no sex differences in the SO group or the E-CO group. Our current results in the SO group were inconsistent with those of the previous study in sesame oil-treated C57BL/6 mice, suggesting that differences in mouse strain might affect the sex difference in D-V length.

For both sexes, D-V length and relative D-V length in the $\mathrm{CO}$ group were comparable to those of the saline group (Figs. $5 \mathrm{C}-5 \mathrm{~F})$. However, male $\mathrm{D}-\mathrm{V}$ length and relative $\mathrm{D}-\mathrm{V}$ length were significantly smaller in the SO group than in the saline group. Although no significant difference in male relative $\mathrm{D}-\mathrm{V}$ length was observed in the E-CO group compared to that of the saline group, male D-V length in the E-CO group was also significantly smaller than in the saline group.

These results showed that sesame oil and $10 \%$ ethanol in corn oil abolished the sex difference in $\mathrm{D}-\mathrm{V}$ length and relative D-V length. Because sesame oil and 10\% ethanol in corn oil reduced $\mathrm{D}-\mathrm{V}$ length and relative $\mathrm{D}-\mathrm{V}$ length in males, but not in females, these oils seemed to affect D-V length particularly in males. However, what only corn oil has no influence on $\mathrm{D}-\mathrm{V}$ length in both sexes suggests that the reduction of male D-V length in E-CO group might be due to the influence of ethanol.

GT Morphology It is reported that there are morphological differences in the GT between sexes and that the male GT forms a tubular urethra within the glans whereas the female urethra remains ventral to the glans. ${ }^{9)}$ In the GT morphology of the saline group, male fetuses formed a tubular urethra and the female urethra opened out ventrally or formed outside of the glans regions (Fig. 6). These developments of the urethra and prepuce on the ventral side may influence the sex difference in D-V length.

These sex differences in the histological sections of the GT were also observed in the other three groups, and the three oil vehicles induced no histological abnormalities in either sex. These results indicated that formation of a tubular urethra during GT development was a robust parameter in addition to AGD and AGI.

\section{CONCLUSION}

In our current study, we evaluated the effects of vehicles themselves on the development of the external genitalia using several parameters: AGD, GT width, D-V length, and GT morphology. Our results indicate that corn oil, sesame oil, and $10 \%$ ethanol in corn oil each potentially have some effect on GT width and D-V length.

Vegetable oils such as corn oil and sesame oil include various kinds of fatty acids. Hilakivi-Clarke et al. reported that a maternal diet high in the corn oil-derived fatty acids increased estrogen levels in pregnant rats, and consequently their offspring showed early puberty onset. ${ }^{10)}$ Therefore, the potential estrogenic action of corn oil and sesame oil might be involved in the reduction of GT width or D-V length in our current results. However, in order to elucidate the point, further investigations are needed to clarify the underlying mechanisms of GT width and D-V length development.

Furthermore, sex differences in GT width and D-V length may depend on the strain of the animal. Therefore, differences in dosing vehicles or animal strains may need to be considered when effects of chemicals on development of external genitalia are evaluated by using GT width or D-V length.

Acknowledgments We gratefully acknowledge the assistance of Mrs. Kyoko Mekada (Laboratory of Hygienic Chemistry and Molecular Toxicology, Gifu Pharmaceutical University) in the collection and analysis of the data. This study was financially supported by a Grant-in-Aid for Scientific Research (B) (24406008) from the Ministry of Education, Culture, Sports, Science and Technology of Japan; by the FY2016 Strategic International Collaborative Research Program (SICORP) from the Japan Science and Technology (JST) Agency; and by the International S\&T Cooperation Program of China (2016YFE0117800).

Conflict of Interest The authors declare no conflict of interest.

\section{REFERENCES}

1) Sato $M$, Wada $K$, Marumo $H$, Nagao $T$, Imai $K$, Ono $H$. Influence of corn oil and diet on reproduction and the kidney in female Sprague-Dawley rats. Toxicol. Sci., 56, 156-164 (2000).

2) Yamasaki K, Sawaki M, Noda S, Takatuki M. Effects of olive, corn, sesame or peanut oil on the body weights and reproductive organ weights of immature male and female rats. Exp. Anim., 50, 173-177 (2001).

3) Miyata K, Yabushita S, Sukata T, Sano M, Yoshino H, Nakanishi T, Okuno Y, Matsuo M. Effects of perinatal exposure to flutamide on sex hormones and androgen-dependent organs in F1 male rats. $J$. Toxicol. Sci., 27, 19-33 (2002).

4) Goto K, Koizumi K, Takaori H, Fujii Y, Furuyama Y, Saika O, Suzuki H, Saito K, Suzuki K. Effects of flutamide on sex maturation and behavior of offspring born to female rats treated during late pregnancy. J. Toxicol. Sci., 29, 517-534 (2004).

5) Clark RL, Anderson CA, Prahalada S, Robertson RT, Lochry EA, Leonard YM, Stevens JL, Hoberman AM. Critical developmental periods for effects on male rat genitalia induced by finasteride, a 5 alpha-reductase inhibitor. Toxicol. Appl. Pharmacol., 119, 34-40 (1993).

6) Bowman CJ, Barlow NJ, Turner KJ, Wallace DG, Foster PM. Effects of in utero exposure to finasteride on androgen-dependent reproductive development in the male rat. Toxicol. Sci., 74, 393-406 (2003).

7) Suzuki H, Suzuki K, Yamada G. Systematic analyses of murine masculinization processes based on genital sex differentiation parameters. Dev. Growth Differ., 57, 639-647 (2015).

8) Gallavan RH Jr, Holson JF, Stump DG, Knapp JF, Reynolds VL. Interpreting the toxicologic significance of alterations in anogenital distance: potential for confounding effects of progeny body weights. Reprod. Toxicol., 13, 383-390 (1999).

9) Seifert AW, Harfe BD, Cohn MJ. Cell lineage analysis demonstrates an endodermal origin of the distal urethra and perineum. Dev. Biol. 318, 143-152 (2008).

10) Hilakivi-Clarke L, Clarke R, Onojafe I, Raygada M, Cho E, Lippman M. A maternal diet high in n-6 polyunsaturated fats alters mammary gland development, puberty onset, and breast cancer risk among female rat offspring. Proc. Natl. Acad. Sci. U.S.A., 94, 9372-9377 (1997). 